\title{
Hypercyclic operators for iterated function systems
}

\author{
Mohammad Salman*(D), Ruchi Das \\ Department of Mathematics, University of Delhi, Delhi-11000\%, India
}

\begin{abstract}
In this paper we introduce and study the notion of hypercyclicity for iterated function systems (IFS) of operators. We prove that for a linear IFS, hypercyclicity implies sensitivity and if an IFS is abelian, then hypercyclicity also implies multi-sensitivity and hence thick sensitivity. We also give some equivalent conditions for hypercyclicity as well as weakly mixing for an IFS of operators.
\end{abstract}

Mathematics Subject Classification (2020). 47A16, 37B05, 37D45, 54H20

Keywords. iterated function systems, hypercyclic operators, sensitive operators, weakly mixing operators

\section{Introduction}

An iterated function system (IFS), introduced by Hutchinson [10], is the semigroup action generated by a finite collection of continuous self maps on a metric space. IFS has a wide range of applications including image processing, stochastic growth models, description of fractals, cf. [5,6]. Chaos theory has been studied extensively in recent years and has sensitive dependence on initial conditions as its key ingredient. In a dynamical system, if there is divergence of close orbits, then there is impossibility regarding prediction of its dynamics and hence sensitivity is the central point of chaos theory.

When we talk about linear dynamical systems it is seen that hypercyclicity directly implies sensitivity as compared to a general dynamical system. Therefore, for chaos theory in linear dynamics, hypercyclicity and sensitivity become more interesting. Moreover, we are interested in time at which the system remains chaotic and hence the study of stronger forms of sensitivity, as initiated by Moothathoo [14], is important and has been studied by many authors for nonautonomous systems as well as for IFS [12,16-18]. In [21], Wu and Zhu have studied different types of chaos including Li-Yorke chaos, Li-Yorke sensitivity, spatio-temporal chaos and distributional chaos and proved their equivalence for bounded operators on Banach spaces. Also, dynamical properties on operators including weakly mixing, hypercyclicity, topological mixing and sensitivity have been studied by many researchers, cf. $[3,4,8,9,11,15]$. Over the last decade, shadowing and chaos for iterated function systems, have gained a lot of attention by many researchers and various interesting results have been obtained $[1,2,7,13,19,20,22]$.

\footnotetext{
*Corresponding Author.

Email addresses: salman25july@gmail.com (M. Salman), rdasmsu@gmail.com (R. Das)

Received: 08.04.2020; Accepted: 26.08.2020
} 
The paper is organized as follows. In Section 2, we give the prerequisites required for remaining sections of the paper. In Section 3, we first introduce the notion of hypercyclic iterated function systems (IFS) of operators and study its equivalence with topological transitivity. We prove that if a linear IFS is hypercyclic, then it is sensitive. Also, if we take IFS to be abelian, then hypercyclicity of the IFS implies multi-sensitivity and hence thick sensitivity of the IFS. Moreover, we give examples/counterexamples of IFS of operators satisfying hypercyclicity. In Section 4, we study weakly mixing of all orders and hypercyclicity for IFS of operators. We give some equivalent conditions for hypercyclicity and weakly mixing for an IFS of operators.

\section{Preliminaries}

Let $X$ be a separable Fréchet space with a translation invariant metric $d$ and $T_{\lambda}: X \rightarrow X$ be a continuous operator, for each $\lambda \in \Lambda$, where $\Lambda$ is a nonempty finite set. Then an iterated function system (IFS) is a semigroup generated by the set consisting of finite sequence of operators and is denoted by $\mathcal{T}=\left\{X ; T_{\lambda} \mid \lambda \in \Lambda\right\}$. An IFS is said to be linear if $X$ in addition have a linear structure and IFS is abelian if the members of $\mathcal{T}$ commute, that is, $T_{\lambda} \circ T_{\nu}=T_{\nu} \circ T_{\lambda}$, for all $\lambda, \nu \in \Lambda$. For any $n \in \mathbb{N}$ and for any $\omega=\left(\lambda_{0}, \lambda_{1}, \lambda_{2}, \ldots\right) \in \Lambda^{\mathbb{Z}^{+}}$, where $\mathbb{Z}^{+}$is the set of all non-negative integers, we consider the $n^{\text {th }}$ iterate of the IFS $\mathcal{T}$ as follows:

$$
T_{\omega}^{n}:=T_{\lambda_{n-1}} \circ T_{\lambda_{n-2}} \circ \cdots \circ T_{\lambda_{0}}, n \in \mathbb{N} \text { and } T_{\omega}^{0}:=\mathrm{id} .
$$

A sequence $\left\{t_{n}\right\}_{n=0}^{\infty}$ is said to be an orbit of the IFS $\mathcal{T}$ if there exists an $\omega=\left(\lambda_{0}, \lambda_{1}\right.$, $\left.\lambda_{2}, \ldots\right) \in \Lambda^{\mathbb{Z}^{+}}$satisfying $t_{n}=T_{\omega}^{n}\left(t_{0}\right)$, for every $n \in \mathbb{Z}^{+}$. In particular, orbit of a point $x \in X$ under $\mathcal{T}$ is given by $\mathscr{O}_{\mathcal{T}}(x)=\left\{T_{\sigma}^{n}(x): n \in \mathbb{Z}^{+}\right.$, for some $\left.\sigma \in \Lambda^{\mathbb{Z}^{+}}\right\}$.

Definition 2.1. An IFS $\mathcal{T}=\left\{X ; T_{\lambda} \mid \lambda \in \Lambda\right\}$ is said to be topologically transitive if for every pair of nonempty open subsets $U$ and $V$ of $X$, there exist an $\omega \in \Lambda^{\mathbb{Z}^{+}}$and an $n \in \mathbb{N}$ such that $T_{\omega}^{n}(U) \cap V \neq \varnothing$.

For any pair of nonempty open subsets $U$ and $V$ of $X$, we denote $N_{\mathcal{T}}(U, V):=\{n \in \mathbb{N}$ : there exists an $\omega \in \Lambda^{\mathbb{Z}^{+}}$such that $\left.T_{\omega}^{n}(U) \cap V \neq \varnothing\right\}$.

Definition 2.2. An IFS $\mathcal{T}=\left\{X ; T_{\lambda} \mid \lambda \in \Lambda\right\}$ is weakly mixing if $\mathcal{T} \oplus \mathcal{T}$ is topologically transitive, where $\mathcal{T} \oplus \mathcal{T}=\left\{X \oplus X ; T_{\lambda} \oplus T_{\nu} \mid \lambda, \nu \in \Lambda\right\}$ and $\left(T_{\lambda} \oplus T_{\nu}\right)(x, y)=\left(T_{\lambda}(x), T_{\nu}(y)\right)$.

For any $x \in X$ and $\epsilon>0$, let $B(x, \epsilon)$ denote the open ball of radius $\epsilon$ with center $x$. For any $\delta>0$ and any nonempty open subset $U$ of $X$, let $N_{\mathcal{T}}(U, \delta)=\{n \in \mathbb{N}$ : $\left.\sup _{\omega \in \Lambda^{\mathbb{Z}^{+}}} \operatorname{diam}\left(T_{\omega}^{n}(U)\right)>\delta\right\}$.

Definition 2.3 ([12]). An IFS $\mathcal{T}=\left\{X ; T_{\lambda} \mid \lambda \in \Lambda\right\}$ is said to have

(1) sensitive dependence on initial conditions (sensitive) if for any nonempty open subset $U$ of $X$, there exists a $\delta>0$ (known as a sensitive constant) such that $N_{\mathcal{T}}(U, \delta) \neq \varnothing$.

(2) multi-sensitivity if for any collection of nonempty open subsets $U_{1}, U_{2}, \ldots, U_{m}$ of $X$, there exists a $\delta>0$ (known as multi-sensitivity constant) such that $\bigcap_{i=1}^{m} N_{\mathcal{T}}\left(U_{i}\right.$, $\delta) \neq \varnothing$.

(3) thick sensitivity if for every nonempty open subset $U$ of $X$, there exists a $\delta>0$ (known as a thickly sensitive constant) such that $N_{\mathcal{T}}(U, \delta)$ is thick, that is, for every $k \in \mathbb{N}$ there exists an $l \in \mathbb{N}$ such that $\{l, l+1, \ldots, l+k\} \subseteq N_{\mathcal{T}}(U, \delta)$. 


\section{Hypercyclic and sensitive operators for linear IFS}

In this section, we introduce and study hypercyclicity for an iterated function system $\mathcal{T}=\left\{X ; T_{\lambda} \mid \lambda \in \Lambda\right\}$ of operators. We prove that if a linear IFS $\mathcal{T}$ is hypercyclic, then it is sensitive. Also, if we take IFS $\mathcal{T}$ to be abelian, then hypercyclicity implies multi-sensitivity and hence thick sensitivity.

Definition 3.1. An IFS $\mathcal{T}=\left\{X ; T_{\lambda} \mid \lambda \in \Lambda\right\}$ of operators is said to be hypercyclic if there exists an $x \in X$, known as hypercyclic vector, such that orbit of $x$ under $\mathcal{T}$ is dense in $X$. The collection of such vectors is denoted by $H C(\mathcal{T})$.

First, we give a characterization for hypercyclic as well as sensitive operators for IFS.

Theorem 3.1. An IFS $\mathcal{T}=\left\{X ; T_{\lambda} \mid \lambda \in \Lambda\right\}$ is hypercyclic if and only if it is topologically transitive. Moreover, $H C(\mathcal{T})$ is a dense $G_{\delta}$-set if $\mathcal{T}$ is hypercyclic or transitive.

Proof. Let $\mathcal{T}$ be hypercyclic and $U, V$ be any pair of nonempty open subsets of $X$. Then by hypercyclicity, there exist a hypercyclic vector $x \in X, \sigma_{1}, \sigma_{2} \in \Lambda^{\mathbb{Z}^{+}}$and $m, n \in \mathbb{N}$ such that $T_{\sigma_{1}}^{m}(x) \in U$ and $T_{\sigma_{2}}^{n}(x) \in V$. If $m<n$, then for $k=(n-m)$, there exists a $\sigma \in \Lambda^{\mathbb{Z}^{+}}$such that $T_{\sigma}^{k}(U) \cap V \neq \varnothing$ and hence $\mathcal{T}$ is topologically transitive in this case. If $m \geq n$, then there exists a set of points $\left\{T_{\omega_{1}}^{k_{1}}(x), T_{\omega_{2}}^{k_{2}}(x), \ldots, T_{\omega_{l}}^{k_{l}}(x)\right\}$, where $\omega_{i} \in \Lambda^{\mathbb{Z}^{+}}$, for $n \leq k_{i} \leq m$ and for $i \in\{1,2, \ldots, l\}$, which is not dense in $V$ because $X$ being Fréchet space has no isolated points. Consequently, there exists a nonempty open subset $V^{\prime}$ of $V$ not containing the set $\left\{T_{\omega_{1}}^{k_{1}}(x), T_{\omega_{2}}^{k_{2}}(x), \ldots, T_{\omega_{l}}^{k_{l}}(x)\right\}$. Again as $x$ is hypercyclic, there exist a $p \in \mathbb{N}$ and a $\sigma_{3} \in \Lambda^{\mathbb{Z}^{+}}$such that $T_{\sigma_{3}}^{p}(x) \in V^{\prime}$. Note that $p>m$ and we get the result as in the previous case. Thus, the IFS $\mathcal{T}$ is transitive.

Conversely, let $\mathcal{T}$ be topologically transitive and $\left\{y_{i}\right\}_{i=1}^{\infty}$ be a countable dense set (which exists because $X$ is separable). Then the open balls of radius $1 / n$ with center $y_{i}$, for $n, i \geq 1$, form a countable base $\left\{U_{j}: j \in \mathbb{N}\right\}$ for $X$. Note that $x \in H C(\mathcal{T})$ if and only if there exist an $m \in \mathbb{Z}^{+}$and a $\sigma \in \Lambda^{\mathbb{Z}^{+}}$such that $T_{\sigma}^{m}(x) \in U_{j}$, for each $j \in \mathbb{N}$. Equivalently, we have

$$
H C(\mathcal{T})=\bigcap_{j=1}^{\infty} \bigcup_{\sigma \in \Lambda^{\mathbb{Z}^{+}}} \bigcup_{m=0}^{\infty}\left(T_{\sigma}^{m}\right)^{-1}\left(U_{j}\right) .
$$

Now, since $T_{\sigma}^{m}$ is continuous and the IFS $\mathcal{T}$ is topologically transitive, therefore $\left(T_{\sigma}^{m}\right)^{-1}\left(U_{j}\right)$, for each $j \in \mathbb{N}$ is open and dense. Thus, by Baire's Category Theorem and (3.1), we get that $H C(\mathcal{T})$ is a dense $G_{\delta}$-set.

Theorem 3.2. Let $\mathcal{T}=\left\{X ; T_{\lambda} \mid \lambda \in \Lambda\right\}$ be a linear IFS such that each $T_{\lambda}$ is a bounded operator on a Banach space $X$. The IFS $\mathcal{T}$ is sensitive if and only if $\sup \left\{\left\|T_{\omega}^{k}\right\|: k \in \mathbb{N}\right\}=$ $\infty$, for some $\omega \in \Lambda^{\mathbb{Z}^{+}}$.

Proof. Let the IFS $\mathcal{T}$ be sensitive, then for any $\epsilon>0$ there exist an $x_{\epsilon} \in B(\Theta, \epsilon)$, an $\omega \epsilon$ $\Lambda^{\mathbb{Z}^{+}}$and an $n_{\epsilon} \in \mathbb{N}$ such that $\left\|T_{\omega}^{n_{\epsilon}}\left(x_{\epsilon}\right)-T_{\omega}^{n_{\epsilon}}(\Theta)\right\|>\delta$, for some $\delta>0$, where $\Theta$ is the zero vector in $X$. Since $\mathcal{T}$ is linear, we get that $\left\|T_{\omega}^{n_{\epsilon}}\left(x_{\epsilon}\right)\right\|>\delta$, where $\left\|x_{\epsilon}\right\|<\epsilon$. Now, as each $T_{\lambda}$ is a bounded operator, $T_{\omega}^{n_{\epsilon}}$ is a bounded operator and hence $\left\|T_{\omega}^{n_{\epsilon}}\left(x_{\epsilon}\right)\right\| \leq\left\|T_{\omega}^{n_{\epsilon}}\right\|\left\|x_{\epsilon}\right\|$. Thus, $\left\|T_{\omega}^{n_{\epsilon}}\right\| \geq\left(\left\|T_{\omega}^{n_{\epsilon}}\left(x_{\epsilon}\right)\right\| /\left\|x_{\epsilon}\right\|\right)>\delta / \epsilon$, for any $\epsilon>0$, which implies that

$$
\sup \left\{\left\|T_{\omega}^{k}\right\|: k \in \mathbb{N}\right\} \geq \sup \left\{\left\|T_{\omega}^{n_{\epsilon}}\right\|: \epsilon>0\right\} \geq \sup \{\delta / \epsilon: \epsilon>0\}=\infty .
$$

Conversely, let $\left.\sup \left\{\left\|T_{\sigma}^{k}\right\|: k \in \mathbb{N}\right\}=\infty\right\}$, for some $\sigma \in \Lambda^{\mathbb{Z}^{+}}$, then for any $r \in \mathbb{N}$, there exist an $n_{r} \in \mathbb{N}$ and a $y_{r} \in X$ such that $\left\|T_{\sigma}^{n_{r}}\left(y_{r}\right)\right\| \geq r$, where $\left\|y_{r}\right\|=1$. Let $y \in X$ and $\xi>0$ be arbitrary and put $n_{\xi}=[2 / \xi]+1$ and $y_{\xi}=y+(\xi / 2) y_{n_{\xi}}$. Therefore, $\left\|y_{\xi}-y\right\|<\xi$ and $\left\|T_{\sigma}^{n_{n_{\xi}}}(y)-T_{\sigma}^{n_{n_{\xi}}}\left(y_{\xi}\right)\right\|=\left\|T_{\sigma}^{n_{n_{\xi}}}\left((\xi / 2) y_{n_{\xi}}\right)\right\|=(\xi / 2)\left\|T_{\sigma}^{n_{n_{\xi}}}\left(y_{n_{\xi}}\right)\right\| \geq(\xi / 2) n_{\xi} \geq 1$. Thus, the IFS $\mathcal{T}$ is sensitive. 
Theorem 3.3. If a linear IFS $\mathcal{T}=\left\{X ; T_{\lambda} \mid \lambda \in \Lambda\right\}$ is hypercyclic, then it is sensitive. Moreover, if IFS $\mathcal{T}$ is abelian, then hypercyclicity of $\mathcal{T}$ implies multi-sensitivity of $\mathcal{T}$.

Proof. For any $x \in X, \epsilon>0$ and $\delta>0$, we have $U=\{y \in X: d(\Theta, y)<\epsilon\}$ and $V=\{y \in X: d(\Theta, y)>\delta\}$ are nonempty open subsets of $X$. Since IFS $\mathcal{T}$ is hypercyclic, therefore there exist an $n \in \mathbb{N}$ and an $\omega \in \Lambda^{\mathbb{Z}^{+}}$such that $T_{\omega}^{n}(U) \cap V \neq \varnothing$. Hence, $T_{\omega}^{n}(z) \in V$, where $z \in U$ which implies that $d\left(T_{\omega}^{n}(z), \Theta\right)>\delta$. Now, since $d$ is translation invariant, therefore $d(x, w)=d(\Theta, z)<\epsilon$, where $w=x+z$ and hence $d\left(T_{\omega}^{n}(x), T_{\omega}^{n}(w)\right)>\delta$. Thus, $\mathcal{T}$ is sensitive.

Next, suppose that $\mathcal{T}$ is abelian and hypercyclic. Let $r_{1}, r_{2} \in X$ be such that $r_{1} \neq r_{2}$ and $d\left(r_{1}, r_{2}\right)=\eta>0$. Let $U_{i}=\left\{x \in X: d\left(x, r_{i}\right)<\eta / 4\right\}$, for $i \in\{1,2\}$ and $V_{1}=\{x \in$ $X: d(x, \Theta)<\xi\}$, for any $\xi>0$. Since IFS $\mathcal{T}$ is topologically transitive, therefore there exist an $l \in \mathbb{N}$, a $y \in U_{1}$ and a $\sigma \in \Lambda^{\mathbb{Z}^{+}}$such that $T_{\sigma}^{l}(y) \in U_{2}$. Moreover, by continuity of $T_{\sigma}^{l}$, we get an open neighborhood $V_{2}$ of $y$ such that $T_{\sigma}^{l}\left(V_{2}\right) \subseteq U_{2}$. Let $U^{\prime}=U_{1} \cap V_{2}$, then $T_{\sigma}^{l}\left(U^{\prime}\right) \subseteq U_{2}$. Also, by linearity, we get that $T_{\sigma}^{l}(\Theta)=\Theta \in V_{1}$ and hence there exists an open neighborhood $W$ of $\Theta$ such that $W \subseteq V_{1}$ with $T_{\sigma}^{l}(W) \subseteq V_{1}$. Again by transitivity, there exist an $m \in \mathbb{N}$, a $\varsigma \in \Lambda^{\mathbb{Z}^{+}}$and a $z \in W$ satisfying $T_{\varsigma}^{m}(z) \in U^{\prime}$ which implies that $\left(T_{\sigma}^{l} \circ T_{\varsigma}^{m}\right)(z) \in U_{2}$. Now, as $\mathcal{T}$ is abelian, therefore $\left(T_{\varsigma}^{m} \circ T_{\sigma}^{l}\right)(z) \in U_{2}$. For $z_{1}=T_{\sigma}^{l}(z) \in V_{1}$ and $z_{2}=z \in V_{1}$, we get that $T_{\varsigma}^{m}\left(z_{1}\right) \in U_{2}$ and $T_{\varsigma}^{m}\left(z_{2}\right) \in U_{1}$. Using triangle inequality, we get that there exist $z_{1}, z_{2} \in X$ and $\delta^{\prime}=\eta / 2>0$ such that

$$
d\left(\Theta, z_{1}\right)<\xi \text { and } d\left(\Theta, z_{2}\right)<\xi \Longrightarrow d\left(T_{\varsigma}^{m}\left(z_{1}\right), T_{\varsigma}^{m}\left(z_{2}\right)\right)>\delta^{\prime} .
$$

Finally, we claim that $\mathcal{T}$ is multi-sensitive with $\delta^{\prime} / 2$ as a constant of multi-sensitivity. Let $x_{1}, x_{2}, \ldots, x_{j} \in X$ be any arbitrary collection of points and $W_{1}, W_{2}, \ldots, W_{j}$ be their respective $\xi$-neighborhoods, for any $\xi>0$. Let $y_{i}=x_{i}+z_{1}$ and $\hat{y}_{i}=x_{i}+z_{2}$, for each $i \in$ $\{1,2, \ldots, j\}$, then using translation invariance of $d$, we get that $d\left(x_{i}, y_{i}\right)<\xi$ and $d\left(x_{i}, \hat{y}_{i}\right)<$ $\xi$, for each $i \in\{1,2, \ldots, j\}$. Moreover using (3.2), we get that $d\left(T_{\varsigma}^{m}\left(y_{i}\right), T_{\varsigma}^{m}\left(\hat{y}_{i}\right)\right)=$ $d\left(\left(T_{\varsigma}^{m}\left(x_{i}\right)+T_{\varsigma}^{m}\left(z_{1}\right)\right),\left(T_{\varsigma}^{m}\left(x_{i}\right)+T_{\varsigma}^{m}\left(z_{2}\right)\right)\right)=d\left(T_{\varsigma}^{m}\left(z_{1}\right), T_{\varsigma}^{m}\left(z_{2}\right)\right)>\delta^{\prime}$, for each $i \in\{1,2, \ldots$, $j\}$. Thus, using the triangle inequality, we get that $d\left(T_{\varsigma}^{m}\left(x_{i}\right), T_{\varsigma}^{m}\left(y_{i}\right)\right)>\delta^{\prime} / 2$ or $d\left(T_{\varsigma}^{m}\left(\hat{y}_{i}\right)\right.$, $\left.T_{\varsigma}^{m}\left(x_{i}\right)\right)>\delta^{\prime} / 2$, where $y_{i}, \hat{y}_{i} \in W_{i}$, for each $i \in\{1,2, \ldots, j\}$ and hence $\mathcal{T}$ is multisensitive.

Corollary 3.2. If an abelian linear IFS $\mathcal{T}=\left\{X ; T_{\lambda} \mid \lambda \in \Lambda\right\}$ is hypercyclic, then it is thickly sensitive.

Proof. As a consequence of Theorem 3.3, it suffices to show that multi-sensitivity implies thick sensitivity. Let $U$ be any nonempty open subset of $X$ and $l \in \mathbb{N}$. Note that by continuity and hypercyclicity of $\mathcal{T}$, we get that $\left(T_{\omega_{j}}^{j}\right)^{-1}(U)$ is a nonempty open subset of $X$, for each $j \in\{0,1, \ldots, l\}$ and for some $\omega_{j} \in \Lambda^{\mathbb{Z}^{+}}$. By multi-sensitivity of IFS $\mathcal{T}$, we get that $\bigcap_{j=0}^{l} N_{\mathcal{T}}\left(T_{\omega_{j}}^{-j}(U), \delta\right)$ is an infinite set, for some $\delta>0$. Therefore, we can choose $n_{l} \geq l$ such that $n_{l} \in \bigcap_{j=0}^{l} N_{\mathcal{T}}\left(T_{\omega_{j}}^{-j}(U), \delta\right)$ which implies that there exists an $\omega^{\prime} \in \Lambda^{\mathbb{Z}^{+}}$ such that $\operatorname{diam}\left(T_{\omega^{\prime}}^{n_{l}}\left(T_{\omega_{j}}^{-j}(U)\right)\right)>\delta$. Thus, there exists a $\sigma_{j} \in \Lambda^{\mathbb{Z}^{+}}$corresponding to each $j \in\{0,1, \ldots, l\}$ such that $\operatorname{diam}\left(T_{\sigma_{j}}^{n_{l}-j}(U)\right)>\delta$ and hence $\left\{n_{l}-l, \ldots, n_{l}-1, n_{l}\right\} \subseteq N_{\mathcal{T}}(U, \delta)$. Consequently, $N_{\mathcal{T}}(U, \delta)$ is thick and IFS $\mathcal{T}$ is thickly sensitive.

The following example justifies that linearity of IFS $\mathcal{T}$ is a necessary condition in Theorem 3.3 and Corollary 3.2.

Example 3.1. Let $S^{1}$ be the unit circle with the arc length metric. Let $\mathcal{T}=\left\{S^{1} ; T_{\lambda} \mid \lambda \in\right.$ $\Lambda\}$, where $\Lambda=\{1,2,3\}$ and $T_{1}\left(e^{i \theta_{1}}\right)=e^{i \theta_{1}}, T_{2}\left(e^{i \theta_{2}}\right)=e^{i\left(\theta_{2}+2 \pi \alpha\right)}$, for a fixed $\alpha \in \mathbb{R} \backslash \mathbb{Q}$ and $T_{3}\left(e^{i \theta_{3}}\right)=e^{i\left(\theta_{3}+2 \pi \gamma\right)}$, for fixed $\gamma \in \mathbb{Q}$. Then the IFS $\mathcal{T}$ is abelian and since for $\lambda_{2}=2 \in \Lambda$, we have $\left(S^{1}, T_{\lambda_{2}}\right)$ is hypercyclic, therefore the IFS $\mathcal{T}=\left\{S^{1} ; T_{\lambda} \mid \lambda \in \Lambda\right\}$ is hypercyclic. But as $T_{\lambda}$ is isometry, for each $\lambda \in \Lambda$, we get that for $e^{i \theta}, e^{i \phi} \in S^{1}$, 
$\left|T_{\omega}^{n}\left(e^{i \theta}\right)-T_{\omega}^{n}\left(e^{i \phi}\right)\right|=\left|e^{i \theta}-e^{i \phi}\right|$, for each $n \in \mathbb{N}$ and for each $\omega \in \Lambda^{\mathbb{Z}^{+}}$. So the IFS $\mathcal{T}$ is not sensitive and hence not thickly sensitive.

Remark 3.1. It is interesting to note that for an abelian IFS $\mathcal{T}=\left\{X ; T_{\lambda} \mid \lambda \in \Lambda\right\}$ if $\Lambda$ is an infinite set and some $\omega \in \Lambda^{\mathbb{Z}^{+}}$is fixed, then Corollary 3.2 need not be true.

Example 3.2. For the space $\mathbb{C}$ of complex numbers; let $H(\mathbb{C})$ be the space of entire functions and the translation operators $T_{\alpha}: H(\mathbb{C}) \rightarrow H(\mathbb{C})$, for $\alpha \neq 0$ be given by $T_{\alpha} f(z)=f(z+\alpha)$. Then $T_{\alpha}$ is an invertible hypercyclic operator [9]. Consider the IFS $\mathcal{T}=\left\{H(\mathbb{C}) ; T_{\lambda} \mid \lambda \in \Lambda\right\}$, where $\Lambda$ is the sequence $(n)_{n \in \mathbb{N}}$, such that $T_{4 n}(f)=$ $T_{4 n-3}(f)=T_{\alpha}^{n}(f)$ and $T_{4 n-2}(f)=T_{4 n-1}(f)=T_{\alpha}^{-n}(f)$, then the IFS $\mathcal{T}$ is abelian. For $\omega=\left\{\lambda_{0}, \lambda_{1}, \lambda_{2}, \ldots\right\} \in \Lambda^{\mathbb{Z}^{+}}$, where $\left\{\lambda_{i}\right\}_{i=0}^{\infty}=i+1$, using hypercyclicity of $\left(H(\mathbb{C}), T_{\alpha}\right)$ and the fact that $\left(T_{\alpha}\right)_{\omega}^{4 n-3}(f)=T_{\alpha}^{n}(f)$, for every $n \in \mathbb{N}$, we get that the IFS $\mathcal{T}$ is hypercyclic. One can verify that $N_{\mathcal{T}}(U, \delta)$, for some $\delta>0$ and for every nonempty open subset $U$ of $H(\mathbb{C})$, is not a thick set because of the fact that $\left(T_{\alpha}\right)_{\omega}^{2 m}(f)=f$, for each $m \in \mathbb{N}$. Thus, the IFS $\mathcal{T}=\left\{H(\mathbb{C}) ; T_{\lambda} \mid \lambda \in \Lambda\right\}$ is hypercyclic but not thickly sensitive.

Remark 3.2. Note that in an IFS $\mathcal{T}=\left\{X ; T_{\lambda} \mid \lambda \in \Lambda\right\}$, if for some $\lambda_{1} \in \Lambda$ the dynamical system $\left(X, T_{\lambda_{1}}\right)$ is hypercyclic or sensitive, then so is the IFS $\mathcal{T}$. However, if we fix some $\omega \in \Lambda^{\mathbb{Z}^{+}}$and the set $\Lambda$ is infinite, then there exists an IFS $\mathcal{T}$ such that $T_{\lambda}$, for each $\lambda \in \Lambda$ is hypercyclic and hence sensitive but the corresponding IFS $\mathcal{T}$ satisfies neither of these properties as seen by the following example.

Example 3.3. Consider the IFS $\mathcal{T}=\left\{H(\mathbb{C}) ; T_{\lambda} \mid \lambda \in \Lambda\right\}$, where $\Lambda$ is the sequence $(n)_{n \in \mathbb{N}}$, as in Example 3.2. Let $T_{2 n-1}(f)=T_{\alpha}(f)$ and $T_{2 n}(f)=T_{\alpha}^{-1}(f)$. Then $\left(H(\mathbb{C}), T_{\alpha}\right)$ is hypercyclic and sensitive. Moreover, we know that an operator $T$ is hypercyclic if and only if $T^{-1}$ is hypercyclic. Thus, $T_{\lambda}$, for each $\lambda \in \Lambda$ is hypercyclic and sensitive. But since the orbit $\mathscr{O}_{\mathcal{T}}(f)=\left\{f, T_{\alpha}(f)\right\}$, for every $f \in H(\mathbb{C})$ is a finite set, therefore the IFS $\mathcal{T}=\left\{H(\mathbb{C}) ; T_{\lambda} \mid \lambda \in \Lambda\right\}$ can be neither hypercyclic nor sensitive.

We end this section by giving an example of a linear abelian IFS $\mathcal{T}=\left\{\ell^{p} ; T_{\lambda} \mid \lambda \in \Lambda\right\}$, for $1 \leq p<\infty$, which is hypercyclic and hence multi-sensitive and thickly sensitive.

Example 3.4. Let $\mathcal{T}=\left\{\ell^{p} ; T_{\lambda} \mid \lambda \in \Lambda\right\}$ be an IFS, where $\Lambda=\{1,2, \ldots, m\}$ and $T_{i}$ : $\ell^{p} \rightarrow \ell^{p}$, for $i \in\{1,2,3, \ldots, m\}$ is given by $T_{i}\left(x_{1}, x_{2}, x_{3}, \ldots\right)=(i+1)\left(x_{2}, x_{3}, x_{4}, \ldots\right)$. It is easy to see that the IFS $\mathcal{T}$ is abelian. Let $U$ and $V$ be any nonempty open subsets of $\ell^{p}$, then we can find $u \in U$ and $v \in V$ such that $u=\left(u_{1}, u_{2}, \ldots, u_{k}, 0,0,0, \ldots\right\}$ and $v=\left(v_{1}, v_{2}, \ldots, v_{k}, 0,0,0, \ldots\right\}$, for some $k \in \mathbb{N}$. For any $n \geq k$ and for any $\sigma \in \Lambda^{\mathbb{Z}^{+}}$, define $w \in \ell^{p}$ by

$$
w_{j}= \begin{cases}u_{j}, & 1 \leq j \leq k \\ \frac{v_{j-n}}{\beta(n, \sigma)}, & n+1 \leq j \leq n+k \\ 0, & \text { otherwise }\end{cases}
$$

where $\beta(n, \sigma)>1$ is such that $\beta(n, \sigma) \rightarrow \infty$ as $n \rightarrow \infty$. Then $\|w-u\|=(\|v\|) /(\beta(n, \sigma))$ $\rightarrow 0$ as $n \rightarrow \infty$. Therefore, $w \in U$ and $T_{\sigma}^{n}(w)=\beta(n, \sigma)\left(w_{n+1}, w_{n+2}, w_{n+3}, \ldots\right)=$ $\left(v_{1}, \ldots, v_{k}, 0,0, \ldots\right)=v$ and hence $T_{\sigma}^{n}(U) \cap V \neq \varnothing$, for every $\sigma \in \Lambda^{\mathbb{Z}^{+}}$and $n \in \mathbb{N}$. Thus, $\mathcal{T}=\left\{\ell^{p} ; T_{\lambda} \mid \lambda \in \Lambda\right\}$, for $1 \leq p<\infty$ is hypercyclic and hence multi-sensitive and thickly sensitive. Moreover, by Theorem 3.2, it can be directly seen that the IFS $\mathcal{T}$ is sensitive as $\sup \left\{\left\|T_{\sigma}^{n}\right\|: n \in \mathbb{N}\right\}=\infty$. 


\section{Hypercyclic and weakly mixing operators for IFS}

In this section, we study weakly mixing of all orders and hypercyclicity of an IFS $\mathcal{T}=\left\{X ; T_{\lambda} \mid \lambda \in \Lambda\right\}$ of operators. Moreover, we give some equivalent conditions for both in an IFS $\mathcal{T}$.

Definition 4.1. An IFS $\mathcal{T}=\left\{X ; T_{\lambda} \mid \lambda \in \Lambda\right\}$ is said to be weakly mixing of order $m(m \geq 2)$ if $\underbrace{T \oplus \cdots \oplus \mathcal{T}}_{m \text {-times }}$ is topologically transitive, that is, for every collection of nonempty open subsets $U_{1}, U_{2}, \ldots, U_{m} ; V_{1}, V_{2}, \ldots, V_{m}$ of $X$, there exist an $n \in \mathbb{N}$ and $\left(\sigma_{1}, \sigma_{2}, \ldots, \sigma_{m}\right) \in$ $\left(\Lambda^{\mathbb{Z}^{+}} \times \cdots \times \Lambda^{\mathbb{Z}^{+}}\right)$such that $\left(T_{\sigma_{1}}^{n} \oplus \cdots \oplus T_{\sigma_{m}}^{n}\right)\left(U_{1} \oplus \cdots \oplus U_{m}\right) \cap\left(V_{1} \oplus \cdots \oplus V_{m}\right) \neq \varnothing$. An IFS is weakly mixing of all orders if it is weakly mixing of order $m$, for all $m \geq 2$.

Remark 4.1. Note that in an IFS $\mathcal{T}=\left\{X ; T_{\lambda} \mid \lambda \in \Lambda\right\}$, if for some $\lambda_{1} \in \Lambda$ the dynamical system $\left(X, T_{\lambda_{1}}\right)$ is weakly mixing of all orders, then so is the IFS $\mathcal{T}$. However, if we fix some $\omega \in \Lambda^{\mathbb{Z}^{+}}$and the set $\Lambda$ is infinite, then there exists an IFS $\mathcal{T}$ such that $T_{\lambda}$, for each $\lambda \in \Lambda$ is weakly mixing of all orders but the corresponding IFS $\mathcal{T}$ is not weakly mixing, which can be seen by Example 3.3.

Theorem 4.1. An abelian IFS $\mathcal{T}=\left\{X ; T_{\lambda} \mid \lambda \in \Lambda\right\}$ is weakly mixing of all orders if and only if for any pair of nonempty open subsets $U$ and $V$ of $X$, we have $N_{\mathcal{T}}(U, U) \cap$ $N_{\mathcal{T}}(U, V) \neq \varnothing$.

Proof. We need to show the sufficiency of the condition. Let $U_{1}, U_{2}, V_{1}, V_{2}$ be any nonempty open subsets of $X$. By given condition, there exist an $n \in \mathbb{N}$ and an $\omega_{1} \in \Lambda^{\mathbb{Z}^{+}}$ such that $T_{\omega_{1}}^{n}\left(U_{1}\right) \cap V_{1} \neq \varnothing$ which implies that $U=U_{1} \cap\left(T_{\omega_{1}}^{n}\right)^{-1}\left(V_{1}\right)$ is a nonempty open subset of $X$. Moreover, by topological transitivity of the IFS $\mathcal{T}$, we get that $\left(T_{\omega_{2}}^{n}\right)^{-1}\left(V_{2}\right)$ is a nonempty open set, for some $\omega_{2} \in \Lambda^{\mathbb{Z}^{+}}$. Therefore, by hypothesis, there exists an $m \in \mathbb{N}$ such that $m \in N_{\mathcal{T}}(U, U) \cap N_{\mathcal{T}}\left(U,\left(T_{\omega_{2}}^{n}\right)^{-1}\left(V_{2}\right)\right)$ and hence there exist $u, v \in U$ and a $\left(\sigma_{1} \times \sigma_{2}\right) \in\left(\Lambda^{\mathbb{Z}^{+}} \times \Lambda^{\mathbb{Z}^{+}}\right)$such that $T_{\sigma_{1}}^{m}(u) \in U$ and $T_{\sigma_{2}}^{m}(v) \in\left(T_{\omega_{2}}^{n}\right)^{-1}\left(V_{2}\right)$ which implies that $T_{\omega_{1}}^{n} \circ T_{\sigma_{1}}^{m}(u) \in V_{1}$ and $T_{\omega_{2}}^{n} \circ T_{\sigma_{2}}^{m}(v) \in V_{2}$. Thus, $T_{\omega}^{m+n}(u) \in V_{1}$ and $T_{\sigma}^{m+n}(v) \in V_{2}$, for some $(\omega \times \sigma) \in\left(\Lambda^{\mathbb{Z}^{+}} \times \Lambda^{\mathbb{Z}^{+}}\right)$and hence we get that $N_{\mathcal{T}}\left(U_{1}, V_{1}\right) \cap N_{\mathcal{T}}\left(U_{1}, V_{2}\right) \neq \varnothing$. Using this condition, the fact that $\mathcal{T}$ is an abelian IFS and proceeding as above one gets that $N_{\mathcal{T}}\left(U_{1}, V_{1}\right) \cap N_{\mathcal{T}}\left(U_{2}, V_{2}\right) \neq \varnothing$ implying that $\mathcal{T}$ is weakly mixing. Since $\mathcal{T}$ is abelian, using similar arguments as given in [8, Theorem 1], we get that the IFS $\mathcal{T}=\left\{X ; T_{\lambda} \mid \lambda \in \Lambda\right\}$ is weakly mixing of all orders.

Theorem 4.2. Let $\mathcal{T}=\left\{X ; T_{\lambda} \mid \lambda \in \Lambda\right\}$ be an IFS, $\left(Y_{n}\right)_{n \in \mathbb{N}}$ be an increasing sequence of subsets of $X$ such that $T_{\lambda}\left(Y_{n}\right) \subseteq Y_{n}$, for each $n \in \mathbb{N}$ and for every $\lambda \in \Lambda$, and $Y=\overline{\bigcup_{n=1}^{\infty} Y_{n}}$. Then, we have:

(i) If the IFS $\left(\mathcal{T} \uparrow_{Y_{n}}\right)$ is hypercyclic, for each $n \in \mathbb{N}$, then the IFS $\mathcal{T}=\left\{Y ; T_{\lambda} \mid \lambda \in \Lambda\right\}$ is hypercyclic.

(ii) If the IFS $\left(\mathcal{T} \uparrow_{Y_{n}}\right)$ is weakly mixing of all orders, for each $n \in \mathbb{N}$, then the IFS $\mathcal{T}=\left\{Y ; T_{\lambda} \mid \lambda \in \Lambda\right\}$ is weakly mixing of all orders.

Proof. $(i)$ Let $U_{1}$ and $U_{2}$ be any pair of nonempty open subsets of $\bigcup_{n=1}^{\infty} Y_{n}$, then there are nonempty open subsets $U_{1}^{\prime}$ and $U_{2}^{\prime}$ of $X$ such that $U_{i}=U_{i}^{\prime} \cap\left(\bigcup_{n=1}^{\infty} Y_{n}\right) \neq \varnothing$, for every $i \in\{1,2\}$. Therefore, there exist $k_{1}, k_{2} \in \mathbb{N}$ such that $U_{i} \cap Y_{k_{i}} \neq \varnothing$, for $i \in\{1,2\}$. We can assume that $k_{1} \leq k_{2}$ and using the fact that $\left(Y_{n}\right)$ is increasing, we get that $Y_{k_{1}} \subseteq Y_{k_{2}}$ and hence $U_{1}^{\prime} \cap Y_{k_{2}} \neq \varnothing$. Now, since $\left(\mathcal{T} \uparrow_{Y_{k_{2}}}\right)$ is hypercylic, therefore there exist an $n \in \mathbb{N}$ and an $\omega=\left\{\omega_{0}, \omega_{1}, \ldots\right\} \in \Lambda^{\mathbb{Z}^{+}}$satisfying $T_{\omega}^{n}\left(U_{1}^{\prime} \cap Y_{k_{2}}\right) \cap\left(U_{2}^{\prime} \cap Y_{k_{2}}\right) \neq \varnothing$. Consequently, we get that $T_{\omega}^{n}\left(U_{1}^{\prime} \cap\left(\bigcup_{n=1}^{\infty} Y_{n}\right)\right) \cap\left(U_{2}^{\prime} \cap\left(\bigcup_{n=1}^{\infty} Y_{n}\right)\right) \neq \varnothing$, that is, $T_{\omega}^{n}\left(U_{1}\right) \cap U_{2} \neq \varnothing$. Thus, the IFS $\mathcal{T}=\left\{\bigcup_{n=1}^{\infty} Y_{n} ; T_{\lambda} \mid \lambda \in \Lambda\right\}$ is hypercyclic and the fact that $\bigcup_{n=1}^{\infty} Y_{n}$ is dense in $Y$ gives that the IFS $\mathcal{T}=\left\{Y ; T_{\lambda} \mid \lambda \in \Lambda\right\}$ is hypercyclic. 
(ii) Let $m \geq 2$ and $U_{1}, U_{2}, \ldots, U_{m} ; V_{1}, V_{2}, \ldots, V_{m}$ be any collection of nonempty open subsets of $\bigcup_{n=1}^{\infty} Y_{n}$, then there exist nonempty open subsets $U_{1}^{\prime}, \ldots, U_{m}^{\prime} ; V_{1}^{\prime}, \ldots, V_{m}^{\prime}$ such that $U_{i}=U_{i}^{\prime} \cap\left(\bigcup_{n=1}^{\infty} Y_{n}\right) \neq \varnothing$ and $V_{i}=V_{i}^{\prime} \cap\left(\bigcup_{n=1}^{\infty} Y_{n}\right) \neq \varnothing$, for each $i \in\{1,2, \ldots, m\}$. Thus, there exist $n_{1}, n_{2}, \ldots, n_{2 m} \in \mathbb{N}$ satisfying $U_{i}^{\prime} \cap Y_{n_{i}} \neq \varnothing$ and $V_{i}^{\prime} \cap Y_{n_{j}} \neq \varnothing$, for each $i \in\{1,2, \ldots, m\}$ and for each $j \in\{m+1, m+2, \ldots, 2 m\}$. Let $k=\max \left\{n_{1}, n_{2}, \ldots, n_{2 m}\right\}$, then we get that $Y_{n_{i}} \subseteq Y_{k}$, for $i \in\{1,2, \ldots, 2 m\}$ and $U_{i}^{\prime} \cap Y_{k} \neq \varnothing$ and $V_{i}^{\prime} \cap Y_{k} \neq \varnothing$, for each $i \in\{1,2, \ldots, m\}$. Now, as $\left(\mathcal{T}\left\lceil_{Y_{k}}\right)\right.$ is weakly mixing of all orders, there exist an $n \in \mathbb{N}$ and a $\left(\sigma_{1}, \sigma_{2}, \ldots, \sigma_{m}\right) \in\left(\Lambda^{\mathbb{Z}^{+}} \times \cdots \times \Lambda^{\mathbb{Z}^{+}}\right)$such that $\left(T_{\sigma_{1}}^{n} \oplus \cdots \oplus T_{\sigma_{m}}^{n}\right)\left(\left(U_{1}^{\prime} \cap Y_{k}\right) \oplus \cdots \oplus\right.$ $\left.\left(U_{m}^{\prime} \cap Y_{k}\right)\right) \cap\left(\left(V_{1}^{\prime} \cap Y_{k}\right) \oplus \cdots \oplus\left(V_{m}^{\prime} \cap Y_{k}\right)\right) \neq \varnothing$. Then proceeding as in part $(i)$, we get that the IFS $\mathcal{T}=\left\{Y ; T_{\lambda} \mid \lambda \in \Lambda\right\}$ is weakly mixing of all orders.

We now give the equivalence of hypercyclic operators in terms of $J(x)$ which is defined as follows:

$$
\begin{array}{r}
J(x)=\left\{y \in X: \text { there exist a strictly increasing sequence }\left\{l_{n}\right\} \subseteq \mathbb{Z}^{+},\right. \\
\left.\left\{x_{n}\right\} \subseteq X, \text { and } \omega_{l_{n}} \in \Lambda^{\mathbb{Z}^{+}} \text {such that } x_{n} \rightarrow x \text { and } T_{\omega_{l_{n}}}^{l_{n}}\left(x_{n}\right) \rightarrow y\right\} .
\end{array}
$$

Theorem 4.3. Let $\mathcal{T}=\left\{X ; T_{\lambda} \mid \lambda \in \Lambda\right\}$ be an IFS, where $X$ is a separable Banach space. Then the following are equivalent:

(1) $\mathcal{T}$ is hypercyclic.

(2) $J(x)=X$, for every $x \in X$.

(3) $F=\{x \in X: J(x)=X\}$ is a dense subset of $X$.

Moreover, if $T_{\lambda}$ is bounded for each $\lambda \in \Lambda$, then the above equivalence is true for $L(x)$, where $L(x)=\left\{y\right.$ : there exist a strictly increasing sequence $\left\{k_{n}\right\} \subseteq \mathbb{Z}^{+}$and $\sigma_{k_{n}} \in \Lambda^{\mathbb{Z}^{+}}$ such that $\left.T_{\sigma_{k_{n}}}^{k_{n}}(x) \rightarrow y\right\}$.

Proof. (1) $\Longrightarrow(2)$. Let $x, y \in X$ be arbitrary. Since by Theorem $3.1, H C(\mathcal{T})$ is a dense $G_{\delta}$-set, therefore there exist a sequence of hypercyclic vectors $x_{n} \subseteq H C(\mathcal{T})$, an increasing sequence of positive integers $l_{n}$ and an $\omega_{l_{n}} \in \Lambda^{\mathbb{Z}^{+}}$such that $x_{n} \rightarrow x$ and $T_{\omega_{l_{n}}}^{l_{n}}\left(x_{n}\right) \rightarrow y$ as $n \rightarrow \infty$ and hence $y \in J(x)$. Thus, $J(x)=X$, for every $x \in X$.

$(2) \Longrightarrow(1)$. Let $\left\{u_{k}\right\}_{k \in \mathbb{N}}$ be a countable dense subset of $X$ and define $\mathcal{U}_{k, l}^{n, \omega}=\{u \in$ $\left.X:\left\|T_{\omega}^{n}(u)-u_{k}\right\|<1 / l\right\}$, for all $k, l \in \mathbb{N}, n \in \mathbb{Z}^{+}$and for some $\omega \in \Lambda^{\mathbb{Z}^{+}}$. Let $v \in X, \epsilon>0$ and $k, l \in \mathbb{N}$ be given, therefore using (2), we get that $J(v)=X$. Consequently, there exist an $u \in X$, a $\sigma \in \Lambda^{\mathbb{Z}^{+}}$and an $n \in \mathbb{N}$ satisfying $\|u-v\|<\epsilon$ and $\left\|T_{\sigma}^{n}(u)-u_{k}\right\|<1 / l$. Thus, $\bigcup_{\omega \in \Lambda^{\mathbb{Z}^{+}}} \bigcup_{n=0}^{\infty} \mathcal{U}_{k, l}^{n, \omega}$ is dense in $X$, for each $k, l \in \mathbb{N}$ and hence in view of Theorem 3.1 and Baire's Category Theorem, we get that $\mathcal{T}$ is hypercyclic.

Clearly, $(2) \Longrightarrow(3)$. We show that $(3) \Longrightarrow(2)$. It suffices to show that if $\left\{x_{n}\right\},\left\{y_{n}\right\}$ are sequences in $X$ such that $x_{n} \rightarrow x, y_{n} \rightarrow y$ and $y_{n} \in J\left(x_{n}\right)$, for every $n \in \mathbb{N}$, then $y \in$ $J(x)$. For $n=1$, there exists an $l_{1} \in \mathbb{N}$ such that $\left\|x_{l_{1}}-x\right\|<1 / 2$ and $\left\|y_{l_{1}}-y\right\|<1 / 2$. Now, as $y_{l_{1}} \in J\left(x_{l_{1}}\right)$, we get a $k_{1} \in \mathbb{N}$, an $\omega_{1} \in \Lambda^{\mathbb{Z}^{+}}$and an $u_{1} \in X$ satisfying $\left\|u_{1}-x_{l_{1}}\right\|<1 / 2$ and $\left\|T_{\omega_{1}}^{k_{1}}\left(u_{1}\right)-y_{l_{1}}\right\|<1 / 2$. Hence, using triangle inequality, we get that $\left\|u_{1}-x\right\|<1$ and $\left\|T_{\omega_{1}}^{k_{1}}\left(u_{1}\right)-y\right\|<1$. Thus, inductively we can find a strictly increasing sequence $\left\{k_{n}\right\} \subseteq \mathbb{Z}^{+}$ and $\left\{u_{n}\right\}$ in $X$ such that $\left\|u_{n}-x\right\|<1 / n$ and $\left\|T_{\omega_{k_{n}}}^{k_{n}}\left(u_{n}\right)-y\right\|<1 / n$, which gives $y \in J(x)$. Therefore, $J(x)=X$, for every $x \in X$.

Finally, we show that the above equivalence is true for $L(x)$ by showing that $J(x)=$ $L(x)$. It suffices to show that $J(x) \subseteq L(x)$. Let $u \in J(x)$, then there exists a strictly increasing sequence $\left\{l_{n}\right\} \subseteq \mathbb{Z}^{+}$, an $\omega_{l_{n}} \in \Lambda^{\mathbb{Z}^{+}}$and a sequence $x_{n} \rightarrow x$ such that $T_{\omega_{l_{n}}}^{l_{n}}\left(x_{n}\right) \rightarrow$ $u$. Since $T_{\lambda}$, for each $\lambda \in \Lambda$ is a bounded operator, therefore we have

$$
\left\|T_{\omega_{l_{n}}}^{l_{n}}(x)-u\right\| \leq\left\|T_{\omega}^{l_{n}}(x)-T_{\omega}^{l_{n}}\left(x_{n}\right)\right\|+\left\|T_{\omega}^{l_{n}}\left(x_{n}\right)-u\right\| \leq A\left\|x_{n}-x\right\|+\left\|T_{\omega}^{l_{n}}\left(x_{n}\right)-u\right\|,
$$

for some $A>0$. Thus, as $n \rightarrow \infty$, we get that $u \in L(x)$ and hence $J(x)=L(x)$, for every $x \in X$. 


\section{Open Problems}

(1) For a linear IFS, is every choatic operator weakly mixing?

(2) Let $\mathcal{T}$ be a linear IFS, where $T_{\lambda}$ is a bounded operator on Banach space $X$, for each $\lambda \in \Lambda$. Can we say that $\mathcal{T}$ is sensitive if and only if $\mathcal{T}^{k}$, for every $k \in \mathbb{N}$ is sensitive if and only if $\mathcal{T}^{m}$, for some $m \in \mathbb{N}$ is sensitive?

(3) Does Theorem 4.3 hold if we take $\mathcal{T}$ to be weakly mixing of all orders and $J^{w}(x)=$ $\left\{y \in X\right.$ : there exist a thick sequence $\left\{l_{n}\right\} \subseteq \mathbb{Z}^{+},\left\{x_{n}\right\} \subseteq X$, and $\omega_{l_{n}} \in \Lambda^{\mathbb{Z}^{+}}$such that $x_{n} \rightarrow x$ and $\left.T_{\omega_{l_{n}}}^{l_{n}}\left(x_{n}\right) \rightarrow y\right\}$ ?

Acknowledgment. The first author is supported by Ministry of Minority Affairs, Government of India, Maulana Azad National Fellowship (F.No. 61-1/2019(SA-III)). The authors are grateful to the anonymous reviewers for careful reading and valuable suggestions.

\section{References}

[1] A.Z. Bahabadi, Shadowing and average shadowing properties for iterated function systems, Georgian Math. J. 22, 179-184, 2015.

[2] A.Z. Bahabadi, On chaos for iterated function systems, Asian-Eur. J. Math. 11, $1850054,2018$.

[3] F. Bayart and É. Matheron, Dynamics of linear operators, 179, Cambridge University Press, Cambridge, 2009.

[4] G. Costakis and A. Manoussos, J-class operators and hypercyclicity, J. Operator Theory, 67, 101-119, 2012.

[5] J.H. Elton and M. Piccioni, Iterated function systems arising from recursive estimation problems, Probab. Theory Related Fields, 91, 103-114, 1992.

[6] B. Forte and E.R. Vrscay, Solving the inverse problem for function/image approximation using iterated function systems. II. Algorithm and computations, Fractals, 2 , 335-346, 1994.

[7] F.H. Ghane, E. Rezaali, and A. Sarizadeh, Sensitivity of iterated function systems, J. Math. Anal. Appl. 469, 493-503, 2019.

[8] K-G. Grosse-Erdmann and A. Peris, Weakly mixing operators on topological vector spaces, Rev. R. Acad. Cienc. Exactas Fís. Nat. Ser. A Mat. RACSAM, 104, 413-426, 2010.

[9] K-G. Grosse-Erdmann and A. Peris, Linear chaos, Springer Science \& Business Media, 2011.

[10] J.E. Hutchinson, Fractals and self-similarity, Indiana Univ. Math. J. 30, 713-747, 1981.

[11] M. Kostić, Chaos for linear operators and abstract differential equations, Nova Science Publishers, New York, 2020.

[12] C. Ma and P. Zhu, A remark on sensitivity and Li-Yorke sensitivity of iterated function systems, Qual. Theory Dyn. Syst. 18, 1-9, 2019.

[13] M. Mohtashamipour and A.Z. Bahabadi, Accessibility on iterated function systems, Georgian Math. J. 28 (1), 117-124, 2021.

[14] T.K.S. Moothathu, Stronger forms of sensitivity for dynamical systems, Nonlinearity, 20, 2115-2126, 2007.

[15] M. Murillo-Arcila and A. Peris, Chaotic behaviour on invariant sets of linear operators, Integral Equations Operator Theory, 81, 483-497, 2015.

[16] M. Salman and R. Das, Furstenberg family and multi-sensitivity in non-autonomous systems, J. Difference Equ. Appl. 25, 1755-1767, 2019. 
[17] M. Salman and R. Das, Multi-transitivity in non-autonomous discrete systems, Topology Appl. 278, 107237, 2020.

[18] M. Salman and R. Das, Sensitivity and property $P$ in non-autonomous systems, Mediterr. J. Math. 17, 128, 2020.

[19] X. Wu, S. Liang, Y. Luo, M. Xin and X. Zhang, A remark on limit shadowing property for iterated function systems, U.P.B. Sci. Bull. Series A, Appl. Math. Phys. 81, 107$114,2019$.

[20] X. Wu, L. Wang and J. Liang, The chain properties and average shadowing property of iterated function systems, Qual. Theory Dyn. Syst. 17, 219-227, 2018.

[21] X. Wu and P. Zhu, On the equivalence of four chaotic operators, Appl. Math. Lett. 25, 545-549, 2012.

[22] X. Zhang, X. Wu, Y. Luo and X. Ma, A remark on limit shadowing for hyperbolic iterated function systems, U.P.B. Sci. Bull., Series A, Appl. Math. Phys. 81, 139-146, 2019 . 